\title{
Singleton-Merten Syndrome: a rare autoimmune disorder caused by a specific IFIH1 mutation
}

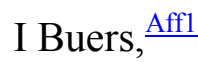

Corresponding Affiliation: Aff1

Y Nitschke, $\stackrel{\text { Affl }}{ }$

RC Hennekam, Aff2

M MacDougall, $\stackrel{\text { Aff3 }}{ }$

$\mathrm{C} \mathrm{Lu}, \stackrel{\operatorname{Aff3}}{ }$

O Mamaeva, Aff3

GI Rice, $\stackrel{\text { Aff4 }}{\text {, }}$

H Erlandsen, Aff3

HG Kehl, $\stackrel{\text { Aff5 }}{\text {, }}$

H Thiele, $\stackrel{\text { Aff } 6}{ }$

P Nürnberg,,$\stackrel{\text { Aff6 }}{ }$

W Höhne,, Aff6

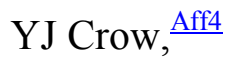

A Feigenbaum, Aff7

F Rutsch, $\stackrel{\text { Affl }}{\underline{1}}$

\begin{tabular}{|c|c|}
\hline ArticleID & 52 \\
\hline ArticleDOI & 10.1186/2194-7791-2-S1-A12 \\
\hline ArticleCitationID & A12 \\
\hline ArticleSequenceNumber & 12 \\
\hline ArticleCategory & Meeting abstract \\
\hline ArticleFirstPage & 1 \\
\hline ArticleLastPage & 3 \\
\hline ArticleHistory & $\begin{array}{l}\text { RegistrationDate } \\
\text { OnlineDate }\end{array}$ \\
\hline
\end{tabular}




\begin{tabular}{|l||l|l||}
\hline \multirow{2}{*}{ ArticleCopyright } & & $\begin{array}{l}\text { Buers et al.2015 } \\
\text { This article is published under license to BioMed Central } \\
\text { Ltd. This is an Open Access article distributed under the } \\
\text { terms of the Creative Commons Attribution License } \\
\text { (http://creativecommons.org/licenses/by/4.0), which permits } \\
\text { unrestricted use, distribution, and reproduction in any } \\
\text { medium, provided the original work is properly cited. }\end{array}$ \\
\hline
\end{tabular}

Aff1

Department of General Pediatrics, Muenster University Children's Hospital, Muenster, Germany

Aff2

Department of Pediatrics, Academic Medical Center, University of Amsterdam, Amsterdam, the Netherlands

Aff3

Institute of Oral Health Research, School of Dentistry, University of Alabama at Birmingham, AL, USA

Aff4

Manchester Academic Health Science Centre, Genetic Medicine, University of Manchester, Manchester, UK

Aff5

Department of Pediatric Cardiology, Muenster University Children's Hospital, Muenster, Germany

Aff6

Cologne Center for Genomics, University of Cologne, Cologne, Germany

Aff7

Division of Clinical and Metabolic Genetics, Hospital for Sick Children, University of San Diego, San

Diego, CA, USA

Abstracts of the 51st Workshop for Pediatric Research

51st Workshop for Pediatric Research

Göttingen, Germany

16-17 April 2015

This supplement has not been sponsored.

Meeting abstracts

\section{Meeting abstract}

Singleton-Merten Syndrome (SMS) is a rare autosomal-dominant disorder characterized by a variety of symptoms including extreme calcifications of the ascending aorta and cardiac valves, dental anomalies (early onset periodontitis, root resorption), psoriasis and widened medullary cavities of the phalanges with focal osteoporosis. Employing whole exome sequencing of five SMS individuals of three families we identified the missense mutation, c.2465G $>$ A (p.Arg822Gln) in the interferon induced with helicase $C$ domain 1 (IFIH1) gene. Additional Sanger sequencing confirmed co-segregation of the mutation with the disorder. IFIH1, encoding melanoma differentiation-associated protein 5 (MDA5), is a member of the RIG-I-like receptor family and functions as a cytoplasmic pattern recognition receptor recognizing viral double stranded RNA (dsRNA). Immunohistochemistry demonstrated the localization of MDA5 in all affected target tissues including heart, skin and cartilage. Functional analysis revealed that the IFIH1 c. $2465 \mathrm{G}>$ A mutation enhanced MDA5 function in interferon beta induction after polyinosinic:polycytidylic acid (poly (I:C)) stimulation in vitro. This indicates that SMS-MDA5 is hyperactive to 
non-self dsRNA. According to additional in vitro studies, interferon signature genes including SIGLEC1 were upregulated in blood and dental cells derived from SMS individuals. Taken together, our data demonstrate that the MDA5 gain-of-function mutation p.Arg822Gln causes the autosomal dominant disorder SMS through dysregulation of the human innate immune response. 